\title{
Successful Motor Evoked Potential Monitoring in Cervical Myelopathy : Related Factors and the Effect of Increased Stimulation Intensity
}

\author{
Hyok Ki Shim, Jae Meen Lee, Dong Hwan Kim, Kyoung Hyup Nam, Byung Kwan Choi, In Ho Han \\ Department of Neurosurgery and Medical Research Institute, Pusan National University Hospital, Pusan National University School of Medi- \\ cine, Busan, Korea
}

Objective : Intraoperative neurophysiological monitoring (IONM) has been widely used during spine surgery to reduce or prevent neurologic deficits, however, its application to the surgical management for cervical myelopathy remains controversial. This study aimed to assess the success rate of IONM in patients with cervical myelopathy and to investigate the factors associated with successful baseline monitoring and the effect of increasing the stimulation intensity by focusing on motor evoked potentials (MEPs).

Methods : The data of 88 patients who underwent surgery for cervical myelopathy with IONM between January 2016 and June 2018 were retrospectively reviewed. The success rate of baseline MEP monitoring at the initial stimulation of $400 \mathrm{~V}$ was investigated. In unmonitorable cases, the stimulation intensity was increased to $999 \mathrm{~V}$, and the success rate final MEP monitoring was reinvestigated. In addition, factors related to the success rate of baseline MEP monitoring were investigated using independent t-test, Wilcoxon rank-sum test, chi-squared test, and Fisher's exact probability test for statistical analysis. The factors included age, sex, body mass index, diabetes mellitus, smoking history, symptom duration, Torg-Pavlov ratio, space available for the cord (SAC), cord compression ratio (CCR), intramedullary increased signal intensity (SI) on magnetic resonance imaging, SI length, SI ratio, the Medical Research Council (MRC) grade, the preoperative modified Nurick grade and Japanese Orthopedic Association (JOA) score.

Results : The overall success rate for reliable MEP response was $52.3 \%$ after increasing the stimulation intensity. No complications were observed to be associated with increased intensity. The factors related to the success rate of final MEP monitoring were found to be SAC $(p<0.001)$, CCR $(p<0.001)$, MRC grade $(p<0.001)$, preoperative modified Nurick grade $(p<0.001)$, and JOA score $(p<0.001)$. The cut-off score for successful MEP monitoring was $5.67 \mathrm{~mm}$ for SAC, $47.33 \%$ for the CCR, 3 points for MRC grade, 2 points for the modified Nurick grade, and 12 points for the JOA score.

Conclusion : Increasing the stimulation intensity could significantly improve the success rate of baseline MEP monitoring for unmonitorable cases at the initial stimulation in cervical myelopathy. In particular, the SAC, CCR, MRC grade, preoperative Nurick grade and JOA score may be considered as the more important related factors associated with the success rate of MEP monitoring. Therefore, the degree of preoperative neurological functional deficits and the presence of spinal cord compression on imaging could be used as new detailed criteria for the application of IONM in patients with cervical myelopathy.

Key Words : Cervical myelopathy · Evoked potentials, Motor · Success rate $\cdot$ Stimulation intensity.

- Received : April 10, 2020 •Revised : May 29, 2020 •Accepted : June 8, 2020

- Address for reprints : In Ho Han

Department of Neurosurgery, Pusan National University Hospital, Pusan National University School of Medicine, 179 Gudeok-ro, Seo-gu, Busan 49241, Korea Tel : +82-51-247-0244, Fax : +82-51-244-0282, E-mail : farlatera|@hanmail.net, ORCID : https://orcid.org/0000-0001-7193-6533

This is an Open Access article distributed under the terms of the Creative Commons Attribution Non-Commercial License (http://creativecommons.org/licenses/by-nc/4.0) which permits unrestricted non-commercial use, distribution, and reproduction in any medium, provided the original work is properly cited. 


\section{INTRODUCTION}

The motor evoked potential (MEP) has recently been proven to be an essential factor in the prevention of permanent neurologic injury in high risk spine surgeries such as those for spinal cord tumor and deformity ${ }^{24)}$. However, the application of MEP monitoring in patients with cervical myelopathy is still controversial due to lack of proven efficacy, high cost, unclear indication, less expertise, and low success rate. In particular, the low success rate of basal MEP is considered a main obstacle in patients with cervical myelopathy. In general, the success rate of baseline MEP recording was reported as 79$98 \%$ in patients without motor weakness or myelopathy ${ }^{8,30,31)}$, however, that of baseline MEP recording was decreased in patients with cervical myelopathy, especially in the lower extremity. Nevertheless, the success rate of baseline MEP monitoring in patients with cervical myelopathy has rarely been reported. Recently, various modifications of stimulation have been proposed to improve the success rate and efficacy of baseline MEP monitoring. Among them, facilitation techniques including double-train stimulation and cutaneous stimulation have been introduced to address the low success rate. However, the usefulness of facilitation techniques is still controversial, particularly in patients with severe myelopathy, due to poor neural pathway conduction. The only reliable and reasonable method, therefore, is to increase the stimulus intensity, yet the success rate of basal MEP monitoring has been rarely reported, and similarly, the efficacy of increasing the stimulus intensity has rarely been investigated in cervical myelopathy. In addition, the preoperative factors affecting the success rate of MEP monitoring and the indications for its application have not yet been clarified.

Therefore, the purpose of this study was to evaluate whether increasing the stimulus intensity would improve the efficacy and success rate of basal MEP monitoring in patients with cervical myelopathy. Furthermore, the preoperative factors affecting the success rate of MEPs were investigated to determine possible indicators for the application of MEP in patients with cervical myelopathy.

\section{MATERIALS AND METHODS}

The study protocol was approved by the Institution Review
Board of Pusan National University Hospital, which waived the requirement for informed consent due to the retrospective nature of this study (IRB No. 1909-015-083).

\section{Patient data}

The data of 88 consecutive patients, who underwent transcranial MEP (TcMEP) during decompression surgery for cervical myelopathy at our hospital between January 2016 and June 2018 were retrospectively reviewed. This study was applied to inclusion criteria only in patients with cervical spondylotic myelopathy, however, patients with severe neuromuscular weakness such as Duchenne muscular atrophy or Rett syndrome and other patients with neurologic deficit due to stroke were excluded, and patients with impaired cord function due to trauma and tumor were also excluded. All patients consented to intraoperative neurophysiological monitoring (IONM) as part of the surgical informed consent process. The age range of the examined patients was 37 to 84 years (mean, $60.76 \pm 12.24)$ and 65 patients were male. The pathological conditions included ossification of the posterior longitudinal ligaments (28 patients), and cervical spondylosis (34 patients), cervical herniated discs (23 patients), and atlantoaxial subluxations (three patients). We evaluated the severity of preoperative cervical myelopathy according to the Medical Research Council (MRC) grade, the modified Nurick grade and the Japanese Orthopedic Association (JOA) score ${ }^{19)}$.

We investigated the factors associated with the success rate of baseline MEP monitoring. These factors included age, sex, body mass index, diabetes mellitus, smoking history, symptom duration, Torg-Pavlov ratio, space available for the cord (SAC), cord compression ratio (CCR), intramedullary increased signal intensity (SI) on magnetic resonance imaging (MRI), SI length, SI ratio, the MRC grade, the preoperative modified Nurick grade and JOA score (Fig. 1). Two experienced spine surgeons performed the measurements using the radiologic images and they were blinded to the patient data.

\section{TcMEP monitoring protocol}

TcMEPs were obtained via transcranial electrode stimulation, using the NIM-ECLIPSE ${ }^{\circledR}$ Monitoring System version 3.5.351 (Medtronic XOMED Inc., Memphis, TN, USA). Transcranial electrical stimulation was delivered by placing an anode (2-cm silver disc) at C3-4 (international 10-20 system for electroencephalography). As constant voltage method, a train 
of 6 pulses (50- $\mu$ s pulse width duration per pulse) with a 2-ms interval was used and these parameters were fixed. A crossscalp stimulating configuration was employed in which C3 was the active stimulating electrode position for left cortical stimulation while $\mathrm{C} 4$ was used for right cortical stimulation. To establish a baseline response, the stimulus intensity was started at $400 \mathrm{~V}$ and gradually increased up to $999 \mathrm{~V}$ at the interval of $200 \mathrm{~V}$ until the MEP generated : $600 \mathrm{~V}, 800 \mathrm{~V}$, and $999 \mathrm{~V}$. The time base was set at $100 \mathrm{~ms}$ and the filter band pass was at 100-5000 Hz. Muscle MEPs were recorded using needle electrodes in four extremities, including anterior tibialis and abductor hallucis. Compound muscle action potential is recorded by inserting two needles 2 to $4 \mathrm{~cm}$ apart in one muscle. Recording was considered successful if a muscle MEP of any amplitude was generated in lower extremities and were in a condition that could be monitored abnormal findings dur- ing surgery. An alert signal was defined as a 50\% sudden drop in any amplitude or loss of CAMP in any muscle.

\section{Somatosensory evoked potential (SSEP) monitor- ing protocol}

SSEP was monitored simultaneously with TcMEP in all cases. Rectangular constant-current stimuli of 500- $\mu$ s duration with intensities up to $30 \mathrm{~mA}$ were applied briefly to either the median nerve at the wrist or the tibial nerve at the ankle at a stimulation rate of $3.9 \mathrm{~Hz}$. The upper extremity SSEP was recorded at $2 \mathrm{~cm}$ behind C3 and the lower extremity SSEP was recorded at $\mathrm{Cz}$ versus $\mathrm{Fz}$, with a band pass from 30 to $3 \mathrm{kHz}$ and average of 200 to 400 sweeps. Any amplitude of SSEP in P37 peak was considered as a successful recording. An alert signal was defined as a $50 \%$ decrease in amplitude or a $10 \%$ increase in response latency or unilateral or bilateral changes
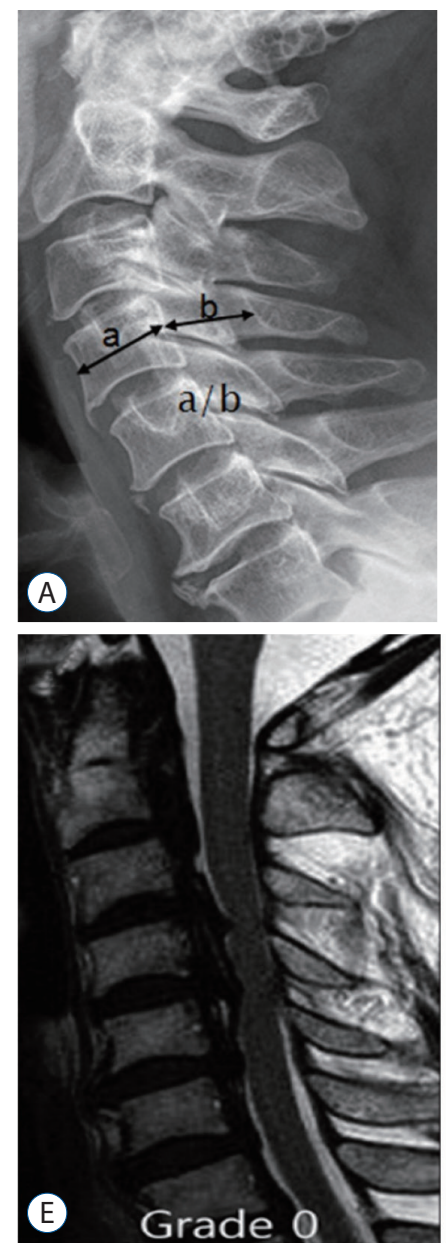
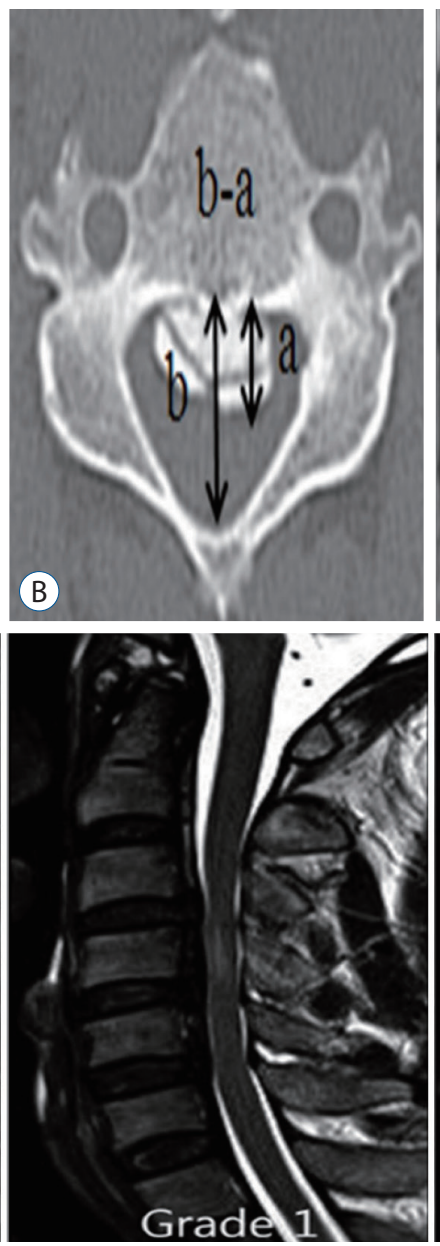
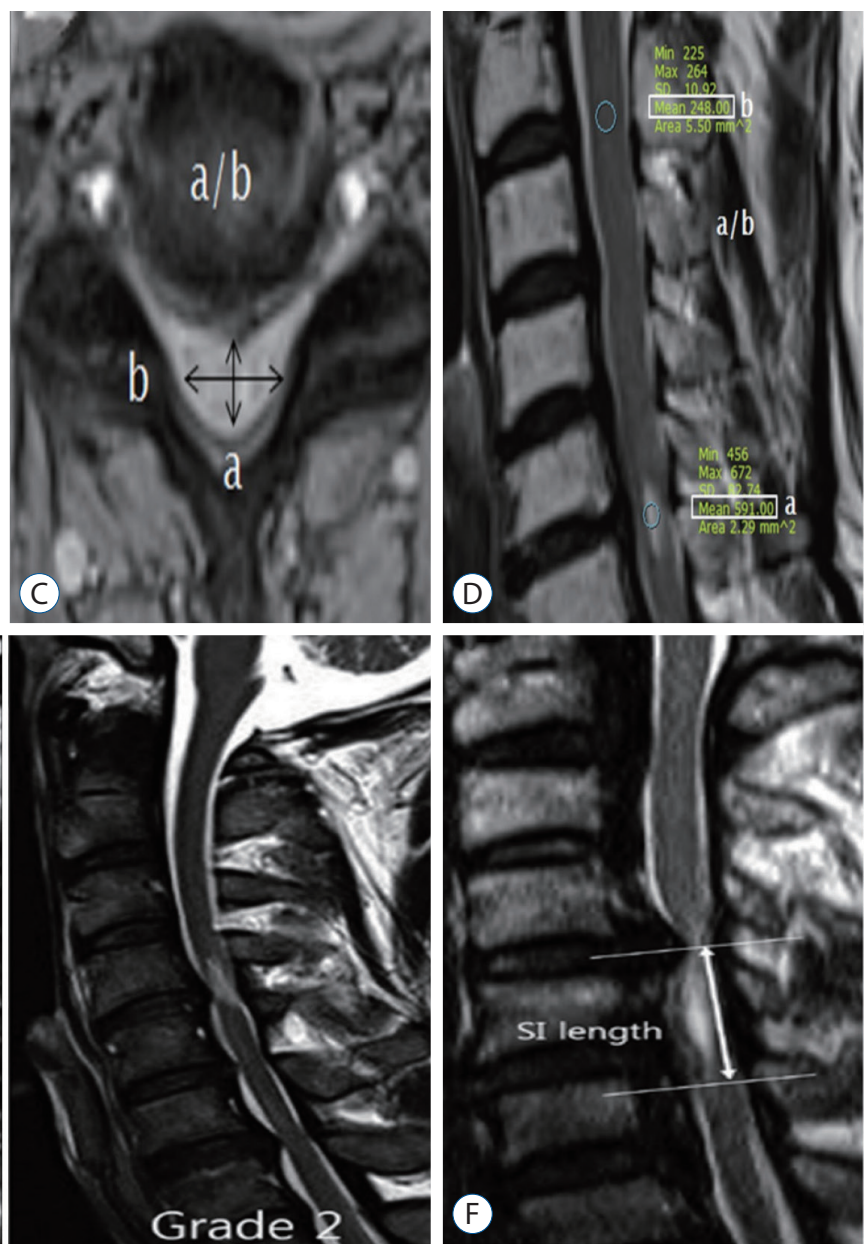

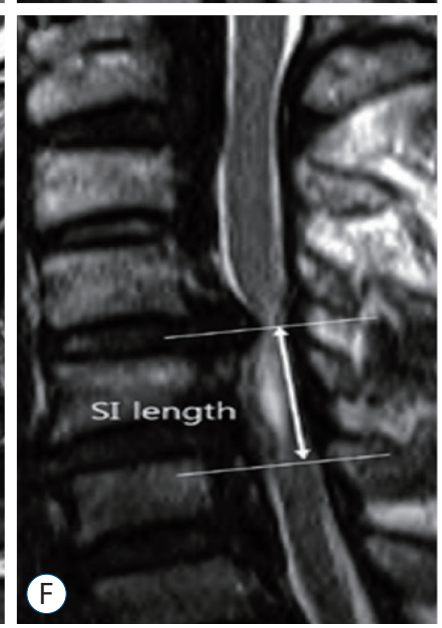

Fig. 1. Measurements of the preoperative radiological factors in cervical myelopathy. A : Torg-Pavlov ratio (a/b). B : Space available for the cord (b-a). C : Cord compression ratio (a/b). D : SI ratio (a/b). E : SI grade. F : SI length. SI : signal intensity. 
in amplitude or latency.

\section{Anesthesia}

General anesthesia was induced by total intravenous anesthesia with propofol (100 to $150 \mu \mathrm{g} / \mathrm{kg} / \mathrm{min}$ ) and remifentanyl $(1 \mu \mathrm{g} / \mathrm{kg})$, avoiding bolus injections whenever possible. A muscle relaxant such as rocuronium was administered only once to facilitate intubation. The mean blood pressure was maintained above $90 \mathrm{mmHg}$.

\section{Statistical analysis}

All analyses were performed using SPSS version 22.0 for
Windows (SPSS Inc., Chicago, IL, USA). The results were expressed as percentage and mean \pm standard deviation. Independent t-test, Wilcoxon rank-sum test, chi-squared test and Fisher's exact probability test were used for the statistical analysis. In addition, inter-rater reliability was calculated from each radiologic measurement analysis of variance, according to the intraclass correlation coefficient and Cohen's kappa. Moreover, receiver operating characteristic curves were constructed for factors and cut-off values determined using MedCalc version 17.4.4 (MedCalc, Ostend, Belgium). A statistical threshold of $p<0.05$ (two-tailed) was considered to be significant.

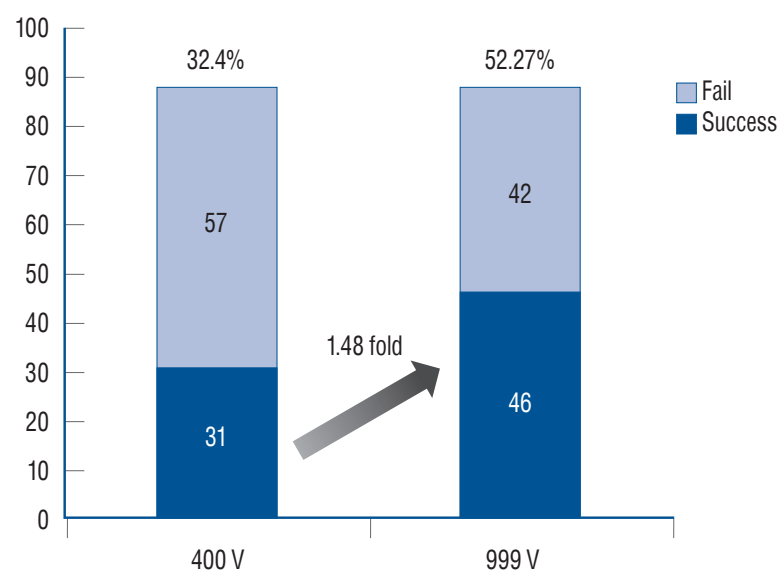

Fig. 2. The graphs show the success rate of motor evoked potentials according to the signal intensity, and total success rate increased by 1.48 folds.

\section{RESULTS}

In our study, 45 patients (51.1\%) had their SSEP measured at the upper brainstem and sensory motor cortex using a scalp electrode to stimulate the posterior tibial nerve and the median nerve. On the other hand, 31 of 88 patients (32.4\%) had their TcMEP recorded in the lower extremities at the initial stimulation of $400 \mathrm{~V}$. We increased the intensity up to $600 \mathrm{~V}$, $800 \mathrm{~V}$, and $999 \mathrm{~V}$. The overall success rate for reliable TcMEP response was $52.27 \%$ (46 patients) after increasing the stimulation intensity in Fig. 2, and each success rate associated with preoperative neurologic factors (MRC grade, Nurick grade, JOA score) are shown in Fig. 3. There was no complication associated with increased intensity, but there were two compli-
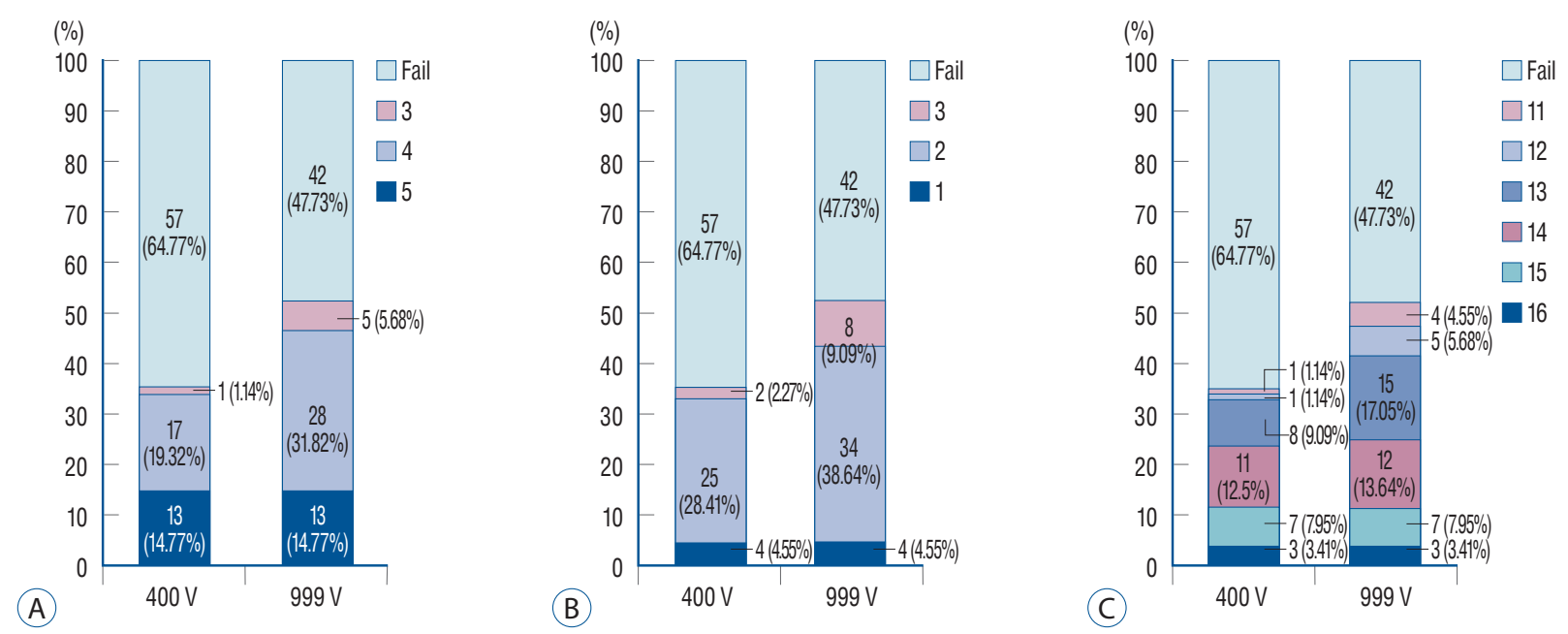

Fig. 3. The graphs show the success rate associated with each preoperative neurologic factors. A : Medical Research Council grade. B : Modified Nurick grade. C: Modified Japanese Orthopedic Association score. 
Table 1. Preoperative factors associated with successful MEP monitoring

\begin{tabular}{|c|c|c|c|c|}
\hline \multirow{2}{*}{ Variable } & \multirow{2}{*}{ Total } & \multicolumn{2}{|c|}{ Success of obtaining MEPs } & \multirow{2}{*}{$p$-value } \\
\hline & & Yes & No & \\
\hline Age (years) & $60.76 \pm 12.24$ & $60.50 \pm 12.35$ & $61.25 \pm 12.56$ & 0.867 \\
\hline Sex, M/F & $65 / 23$ & $33 / 13$ & $32 / 10$ & 0.635 \\
\hline BMl & $24.98 \pm 3.12$ & $24.92 \pm 3.11$ & $25.05 \pm 3.16$ & 0.852 \\
\hline $\mathrm{DM}, \mathrm{Y} / \mathrm{N}$ & $28 / 60$ & $12 / 34$ & $16 / 26$ & 0.227 \\
\hline Smoking, Y/N & $37 / 51$ & $21 / 25$ & $16 / 26$ & 0.473 \\
\hline Symptom duration & $35.21 \pm 41.86$ & $33.14 \pm 36.60$ & $39.00 \pm 51.73$ & 0.703 \\
\hline TPR & $0.42 \pm 0.18$ & $0.47 \pm 0.20$ & $0.31 \pm 0.76$ & 0.345 \\
\hline SAC & $5.79 \pm 1.45$ & $6.39 \pm 1.24$ & $4.68 \pm 1.14$ & $<0.001^{*}$ \\
\hline$C C R$ & $46.01 \pm 8.20$ & $49.18 \pm 7.35$ & $42.54 \pm 7.73$ & $<0.001^{*}$ \\
\hline SI on T1WI MRI, 0/1/2 & $1 / 34 / 53$ & $1 / 20 / 25$ & $0 / 14 / 28$ & 0.061 \\
\hline SI length & $22.63 \pm 12.65$ & $20.54 \pm 12.67$ & $24.93 \pm 12.37$ & 0.104 \\
\hline SI ratio & $1.39 \pm 0.55$ & $1.38 \pm 0.45$ & $1.42 \pm 0.65$ & 0.073 \\
\hline MRC grade & $3.48 \pm 0.98$ & $4.13 \pm 0.64$ & $2.76 \pm 0.77$ & $<0.001^{*}$ \\
\hline Preoperative modified Nurick grade & $2.38 \pm 0.88$ & $1.93 \pm 0.71$ & $2.86 \pm 0.78$ & $<0.001^{*}$ \\
\hline Preoperative modified JOA score & $11.92 \pm 2.24$ & $13.48 \pm 1.62$ & $10.21 \pm 1.44$ & $<0.001^{*}$ \\
\hline
\end{tabular}

Values are presented as the mean \pm standard error unless otherwise indicated. ${ }^{*} p<0.05$. MEP : motor evoked potential, $\mathrm{M}:$ male, F : female, BMI : body mass index, DM : diabetes mellitus, Y : yes, N : no, TPR : Torg-Pavlov ratio, SAC : space available for the cord, CCR : cord compression ratio, SI : signal intensity, TIWI : T1-weighted imaging, MRI : magnetic resonance imaging, MRC : Medical Research Council, JOA : Japanese Orthopedic Association

cations secondary to surgery, C5 palsy after laminoplasty and dysphagia after anterior cervical discectomy and fusion.

A total of five neurophysiologic monitoring alerts were detected. A total of three patients (6.7\%) had SSEP alerts related to banded arm malposition, hypotension and tethering nerve root due to laminoplasty, respectively. On the other hands, TcMEP alerts were detected in two patients (4.3\%) who had neurologic deficits after surgery. The sensitivity rates of SSEP and TcMEP alerts for detecting impending neurologic injury were $50 \%$ and $100 \%$, respectively, and the specificity rates were $97.7 \%$ and $100 \%$, respectively. The positive predictive value rates were $33.3 \%$ and $100 \%$, respectively, while the negative predictive value rates were $98.8 \%$ and $100 \%$, respectively.

As shown in Table 1, SAC, CCR, the MRC grade, the preoperative modified Nurick grade, and the JOA score $(p<0.05)$ were the only variables with a statistically significant association with the success of obtaining MEPs in patients who had cervical myelopathy. That is, patients with a wide SAC, low CCR, low modified Nurick grade, high MRC grade and JOA score had a higher success rate of obtaining MEPs than those with inverse variables.

The factors associated with the overall success rate of MEP monitoring were SAC $(p<0.001)$, CCR $(p<0.001)$, the MRC grade $(p<0.001)$, the preoperative modified Nurick grade $(p<0.001)$ and the JOA score $(p<0.001)$. The cut-off scores for successful MEP monitoring in terms of SAC, CCR, MRC grade, the preoperative modified Nurick grade and the JOA score were $5.67 \mathrm{~mm}$ (sensitivity, 76.09\%; specificity, 92.86\%), $47.33 \%$ (sensitivity, $67.39 \%$; specificity, $76.19 \%$ ), 3 points (sensitivity, $82.61 \%$; specificity, $80.95 \%$ ), 2 points (sensitivity, $78.26 \%$; specificity, $66.67 \%$ ), and 12 points (sensitivity, 84.78\%; specificity, 97.62\%), respectively (Fig. 4). There was no difference in the reliability analysis between the two surgeons (Table 2).

\section{DISCUSSION}

In our experiences of MEP application in patients with cervical myelopathy, the success rate of basal MEP monitoring was very low at a stimulation intensity of $200 \mathrm{~V}$, which is considered an adequate initial intensity. Therefore, we set the initial stimulus intensity at $400 \mathrm{~V}$. Nevertheless, the success rate was very low; the application of MEP monitoring limited in 


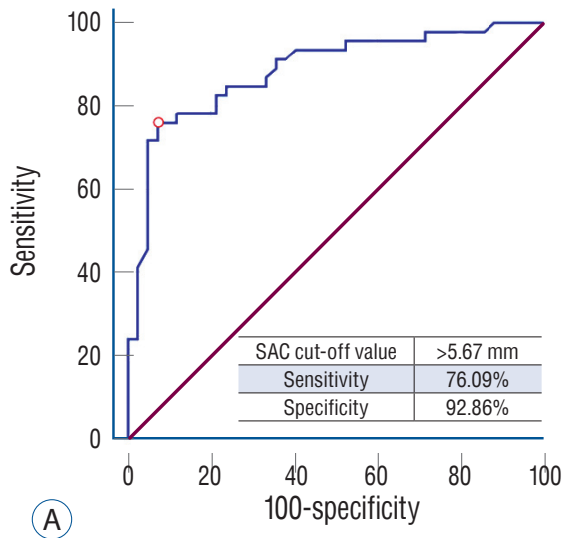

(A)

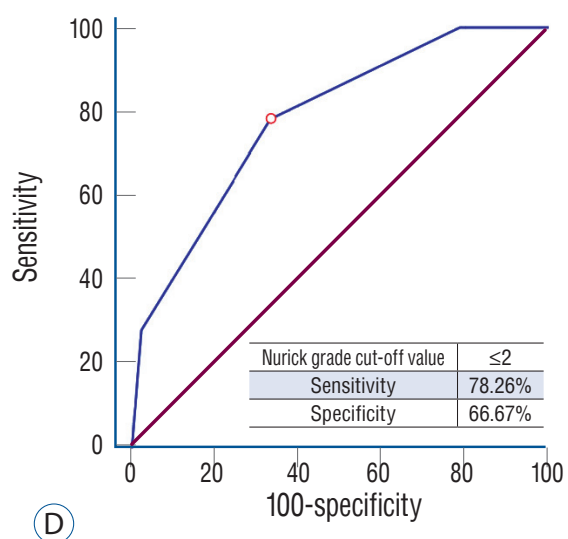

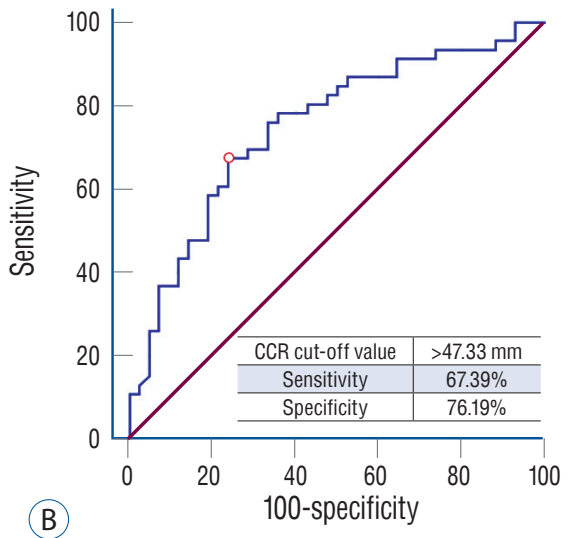

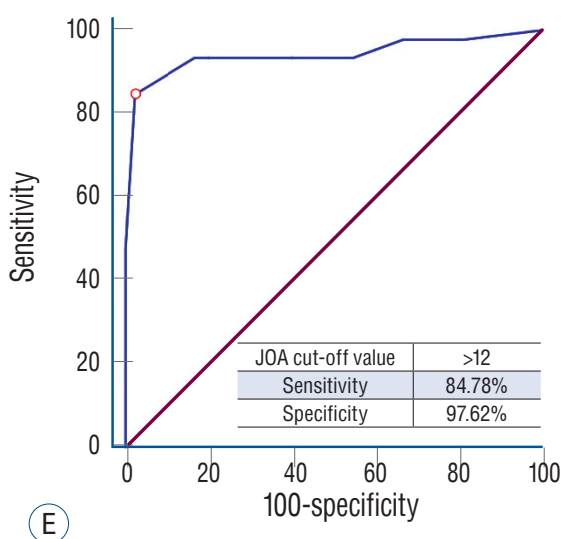

(E)

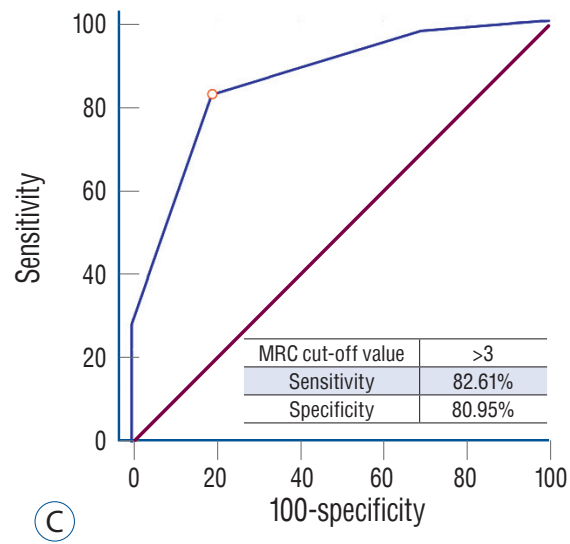

(C)

Fig. 4. The graphs represent the receiver operating characteristics $(R O C)$ curves of important relevant factors for success of obtaining motor evoked potentials (MEPs). A : Space available for the cord (SAC). B : Cord compression ratio (CCR). C : Medical Research Council (MRC) grade. D : Modified Nurick grade. E: Modified Japanese Orthopedic Association (JOA) score.

Table 2. Inter-rater reliability for radiologic and neurological measurements of cervical myelopathy

\begin{tabular}{lcc}
\hline & ICC or Cohen's kappa & 95\% Cl \\
\hline TPR $^{*}$ & 0.933 & $0.907-0.960$ \\
SAC $^{*}$ & 0.901 & $0.847-0.934$ \\
CCR $^{*}$ & 0.946 & $0.918-0.965$ \\
SI on T1WI MRI (0/1/2) & $0.959-0.995$ \\
SI length* $^{\dagger}$ & 0.897 & $0.891-0.953$ \\
SI ratio* $^{*}$ & 0.928 & $0.806-0.917$ \\
MRC grade $^{\dagger}$ & 0.873 & $0.914-0.946$ \\
Preoperative modified Nurickgrade $^{\dagger}$ & 0.912 & $0.921-1.012$ \\
Preoperative modified JOA score $^{\dagger}$ & 0.967 & $0.962-0.983$ \\
\hline
\end{tabular}

*ICC for continuous variables. ${ }^{\dagger}$ Cohen's kappa coefficient for categorical variables, $95 \% \mathrm{Cl}$. ICC : intraclass correlation coefficient, $\mathrm{Cl}$ : confidence interval, TPR : Torg-Pavlov ratio, SAC : space available for the cord, CCR : cord compression ratio, SI : signal intensity, TIWI : T1-weighted imaging, MRI : magnetic resonance imaging, MRC : Medical Research Council, JOA : Japanese Orthopedic Association

patients with cervical myelopathy because of possible dysfunction of the ventral motor spinal tract. In unmonitorable cases, a stimulation modification such as facilitating the tech- nique of MEP could improve the success rate of baseline MEP recording. A facilitating the technique of stimulation such as modifying the intensity of the stimulation current, duration, 
and interval between the stimulation is a basic method to improve the success rate of TcMEP monitoring. Thus, increasing the stimulus intensity appears to be the most effective method for improving the success rate in patients with cervical myelopathy. However, one should keep in mind that high-voltage stimulation over $1000 \mathrm{~V}$ can cause complications such as tongue bite, seizure, and scalp burn, limiting the frequent use of increasing stimulation intensity.

Many investigators have demonstrated the usefulness of IONM during cervical spine surgery. Clark et al. ${ }^{11)}$ and Eggspuehler et al. ${ }^{13)}$ reported high sensitivity and specificity of predicting postoperative deficits in patients undergoing operative procedures for degenerative cervical myelopathy with the use of IOMN. Similarly, others showed the effectiveness of IONM in intra and postoperative neurologic deficit following cervical spine surgery ${ }^{18,23)}$. However, there is a continuing debate on the utility of methods for predicting and mitigating postoperative neurological defects in patients with cervical myelopathy that are considered cost-effective. Unfortunately, thus far, the management of IONM alerts is still inconsistent in the spinal medical community, which may partially explain the lack of evident improvement in the overall incidence of neurological events ${ }^{5,26,34)}$. Furthermore, the overall cost-effectiveness of IONM in spine surgery remains controversial in the current literature ${ }^{3,27,28)}$.

Despite several conflicting opinions, the IONM to be applied in cervical myelopathy patients consist of the following (in order of benefits) : MEP, spinal cord evoked potential, SSEP, and electromyography. Although the MEP has been found to be a significant parameter for predicting outcomes of cervical myelopathy surgery, there is no evidence that it is more valuable than clinical parameters ${ }^{40)}$. Several studies on MEP monitoring in cervical myelopathy have reported its efficacy in terms of sensitivity and specificity during spine surgery ${ }^{12,22,32,33)}$. However, the initial successful monitoring of MEP should precede evaluation of its sensitivity and specificity in patients with cervical myelopathy. Although the success rate of basal MEP monitoring is practically low in patients with cervical myelopathy, there has been no study on the success rate, efficacy of increasing stimulation intensity, and preoperative factors that could affect the success rate of MEP until now ${ }^{20,26,39)}$.

In this study, the success rate of basal MEP monitoring was $32.4 \%$ at a stimulation intensity of $400 \mathrm{~V}$. The success rate increased in proportion to the increase in stimulus intensity, which finally reached up to $52.27 \%$ at an intensity of $999 \mathrm{~V}$.

Lee et al. ${ }^{25)}$ showed the success rate of recording MEP and SSEP according to the MRC grade of motor function in IONM application for spine surgery : MEP 100\% and SSEP $85.4 \%$ in normal MRC grade 5 patients without myelopathy. Also, Chen et al. ${ }^{8)}$ reported that the success rate of lower extremity MEP monitoring was $78.9 \%$ in patients without motor weakness compared with $39.1 \%$ in patients with motor weakness. Therefore, our results showed that the success rate of MEP monitoring in patients with cervical myelopathy is indeed low in comparison with that of patients with normal cord function. In other words, the functional grades represent an independent predictor for the low success rate of MEP recordings. In addition, MEP monitoring could be ineffective in approximately $50 \%$ of patients with low functional grades.

Among many preoperative factors, SAC, CCR, the MRC grade, the preoperative modified Nurick grade and the JOA score were found to be influential factors affecting successful monitoring in patients with cervical myelopathy. The results indicate that the preoperative neurologic state and the extent of cervical cord compression are very important parameters for successful monitoring. Based on their cut-off value, successful monitoring is expected when the modified Nurick grade is less than 3 points, the modified JOA score is over 12 points, the MRC grade is over 3 points, and the CCR and SAC are over $47.33 / 5.15 \mathrm{~mm}$ on MRI. We suggest that these values could be a possible guideline for MEP monitoring in patients with cervical myelopathy. Certainly, MEP monitoring can be successful in patients satisfying these reference point values. Wang et al. ${ }^{37)}$ reported that an improvement in the preexisting intraoperative MEP wave can predict better outcomes for early- and long-term neurologic recovery in patients with cervical myelopathy. Although the MEP can be ineffective in patients having a score below 12 points on the modified JOA score, in this study, the increasing simulation intensity method enabled successful MEP monitoring of about $4.55 \%$ (three patients) at 11 points. In terms of cost-effectiveness, IOMN application may be recommended for patients with cervical myelopathy having a score of 12 or more in accordance with the cut-off value of the modified JOA score. However, in patients with a high risk of neurologic deficit during surgery (score of at least 11 points), better clinical outcomes can be expected if IOMN is carefully applied through increasing the simulation intensity in combination with double-train ${ }^{21)}$ or high frequency-train 
stimulus ${ }^{6,17)}$. Besides the method of increasing simulation intensity, the concepts of other facilitating techniques are that a preconditioning pulse train preceding a multiple transcranial electrical stimulus leads to a larger MEP response. Another unique aspect of this study is that, even though several studies have shown that the SI (strength, length, grade) of T2-weighted imaging was not statistically significant in terms of the success rate of MEP, its importance in myelopathy severity and surgical outcomes has been demonstrated ${ }^{2,10,15,38)}$. However, in consideration of the statistic value, it still appears to be relatively correlated compared to other factors, also as it increased, the success rate of MEP tended to decrease.

The application of MEP monitoring may be difficult in most patients with cervical myelopathy. We speculate that several studies on MEP monitoring in patients with cervical myelopathy excluded the unmonitorable cases in terms of initial MEP. Thus, we recommend that the rate of unmonitorable cases should be considered prior to evaluating the efficacy or cost-effectiveness of MEP monitoring in patients with cervical myelopathy.

This study has several limitations. First, the sample size of this study is small; hence, a larger population of patients could have provided more significant statistical results. Second is that our study did not include additional non-neurological related factors that can probably affect the wave forms such as history of steroid injection, past surgical history, and also very specific anesthetic components (heart rate, temperature, partial pressure of alveolar carbon dioxide) ${ }^{4}$. Third, this study only reflects the success rate of MEP monitoring and does not include a control group of patients without cervical myelopathy. Therefore, a more thorough analysis could be achieved in a prospective study with a larger number of patients. Considering the findings of previous studies including ours, it is suggested that the surgical outcome and prognostic factors consist of the following patient clinical characteristics : age at surgery, sex, duration of symptoms, preoperative JOA score, radiological findings, spinal cord evoked potential type, surgical procedure, and follow-up period duration ${ }^{9,35,36)}$.

Some controversy remains about the overall efficacy of surgery for cervical myelopathy ${ }^{7,14)}$ although recent data have associated IONM with postoperative clinical outcome benefits ${ }^{1,16,29)}$. Further research that utilizes larger samples, comparable and efficient stimulation protocols, and standardized outcome measures for longer follow-up periods may shed some light on this matter.

\section{CONCLUSION}

Increasing the stimulation intensity could significantly improve the success rate of baseline MEP monitoring for unmonitorable cases at the initial stimulation in cervical myelopathy. In particular, SAC, CCR, the MRC grade, the preoperative Nurick grade and the JOA score may be considered as the more important factors associated with the success rate of MEP monitoring. Therefore, the degree of preoperative neurological functional deficits and spinal cord compression on imaging could be used as new detailed criteria for the indication of IONM in patients with cervical myelopathy.

\section{CONFLICTS OF INTEREST}

No potential conflict of interest relevant to this article was reported.

\section{INFORMED CONSENT}

This type of study does not require informed consent.

\section{AUTHOR CONTRIBUTIONS}

\author{
Conceptualization : IHH \\ Data curation : HKS, DHK \\ Formal analysis : HKS \\ Methodology : JML \\ Project administration : IHH \\ Visualization: HKS, KHN \\ Writing - original draft : HKS \\ Writing - review \& editing: HKS, IHH, BKC
}

\section{ORCID}

$\begin{array}{ll}\text { Hyok Ki Shim } & \text { https://orcid.org/0000-0001-5118-4098 } \\ \text { Jae Meen Lee } & \text { https://orcid.org/0000-0002-5708-1644 }\end{array}$ 
Dong Hwan Kim https://orcid.org/0000-0001-8982-7917

Kyoung Hyup Nam https://orcid.org/0000-0002-3749-4660

Byung Kwan Choi https://orcid.org/0000-0001-5244-4592

In Ho Han https://orcid.org/0000-0001-7193-6533

\section{References}

1. Appel S, Biron T, Goldstein K, Ashkenazi E : Effect of intra- and extraoperative factors on the efficacy of intraoperative neuromonitoring during cervical spine surgery. World Neurosurg 123 : e646-e651, 2019

2. Avadhani A, Rajasekaran S, Shetty AP : Comparison of prognostic value of different MRI classifications of signal intensity change in cervical spondylotic myelopathy. Spine J 10 : 475-485, 2010

3. Ayoub C, Zreik T, Sawaya R, Domloj N, Sabbagh A, Skaf G : Significance and cost-effectiveness of somatosensory evoked potential monitoring in cervical spine surgery. Neurol India 58 : 424-428, 2010

4. Benuska J, Plisova M, Zabka M, Horvath J, Tisovsky P, Novorolsky K : The influence of anesthesia on intraoperative neurophysiological monitoring during spinal surgeries. Bratisl Lek Listy 120 : 794-801, 2019

5. Bose B, Sestokas AK, Schwartz DM : Neurophysiological monitoring of spinal cord function during instrumented anterior cervical fusion. Spine J 4 : 202-207, 2004

6. Calancie B, Harris W, Brindle GF, Green BA, Landy HJ : Threshold-level repetitive transcranial electrical stimulation for intraoperative monitoring of central motor conduction. J Neurosurg 95(2 Suppl) : 161-168, 2001

7. Charalampidis A, Jiang F, Wilson JR, Badhiwala JH, Brodke DS, Fehlings MG : The use of intraoperative neurophysiological monitoring in spine surgery. Global Spine J 10(1_suppl) : 104S-114S, 2020

8. Chen X, Sterio D, Ming X, Para DD, Butusova M, Tong T, et al. : Success rate of motor evoked potentials for intraoperative neurophysiologic monitoring: effects of age, lesion location, and preoperative neurologic deficits. J Clin Neurophysiol 24 : 281-285, 2007

9. Cho YE, Shin JJ, Kim KS, Chin DK, Kuh SU, Lee JH, et al. : The relevance of intramedullary high signal intensity and gadolinium (Gd-DTPA) enhancement to the clinical outcome in cervical compressive myelopathy.

Eur Spine J 20 : 2267-2274, 2011

10. Choi JH, Shin JJ, Kim TH, Shin HS, Hwang YS, Park SK : Does intramedullary signal intensity on MRI affect the surgical outcomes of patients with ossification of posterior longitudinal ligament? J Korean Neurosurg Soc 56 : 121-129, 2014

11. Clark AJ, Safaee M, Chou D, Weinstein PR, Molinaro AM, Clark JP 3rd, et al. : Comparative sensitivity of intraoperative motor evoked potential monitoring in predicting postoperative neurologic deficits: nondegenerative versus degenerative myelopathy. Global Spine J 6 : 452-458, 2016

12. Cole T, Veeravagu A, Zhang M, Li A, Ratliff JK : Intraoperative neuromonitoring in single-level spinal procedures: a retrospective propensity score-matched analysis in a national longitudinal database. Spine (Phila
Pa 1976) 39 : 1950-1959, 2014

13. Eggspuehler A, Sutter MA, Grob D, Jeszenszky D, Porchet F, Dvorak J : Multimodal intraoperative monitoring (MIOM) during cervical spine surgical procedures in 246 patients. Eur Spine J 16(Suppl 2) : 209-215, 2007

14. Fouyas IP, Statham PF, Sandercock PA : Cochrane review on the role of surgery in cervical spondylotic radiculomyelopathy. Spine (Phila Pa 1976) $27: 736-747,2002$

15. Geng T, Zhu X, Xu C : Changes of T2WI high signal of spinal magnetic resonance imaging correlated with the 1-year postoperative improvement rate in patients with cervical spondylotic myelopathy. Int J Clin Exp Med 12 : 13472-13479, 2019

16. Gerling MC, Radcliff K, Isaacs R, Bianco K, Jalai CM, Worley NJ, et al. : Two-year results of the prospective spine treatment outcomes study: an analysis of complication rates, predictors of their development, and effect on patient derived outcomes at 2 years for surgical management of cervical spondylotic myelopathy. World Neurosurg 106 : 247-253, 2017

17. Halawa I, Reichert K, Sommer M, Paulus W : Increasing pulse widths and intensity increase the efficacy of high frequency rTMS in inducing excitatory aftereffects. bioRxiv, 2019 [Epub ahead of print]

18. Hilibrand AS, Schwartz DM, Sethuraman V, Vaccaro AR, Albert TJ : Comparison of transcranial electric motor and somatosensory evoked potential monitoring during cervical spine surgery. J Bone Joint Surg Am 86 : 1248-1253, 2004

19. Hirabayashi K, Miyakawa J, Satomi K, Maruyama T, Wakano K : Operative results and postoperative progression of ossification among patients with ossification of cervical posterior longitudinal ligament. Spine (Phila Pa 1976) 6 : 354-364, 1981

20. Ito Z, Matsuyama Y, Shinomiya K, Ando M, Kawabata S, Kanchiku T, et al. : Usefulness of multi-channels in intraoperative spinal cord monitoring: multi-center study by the Monitoring Committee of the Japanese Society for Spine Surgery and Related Research. Eur Spine J 22 : 18911896, 2013

21. Journée $H L$, Polak HE, de Kleuver M, Langeloo DD, Postma AA : Improved neuromonitoring during spinal surgery using double-train transcranial electrical stimulation. Med Biol Eng Comput 42 : 110-113, 2004

22. Khan MH, Smith PN, Balzer JR, Crammond D, Welch WC, Gerszten P, et al. : Intraoperative somatosensory evoked potential monitoring during cervical spine corpectomy surgery: experience with 508 cases. Spine (Phila Pa 1976) 31 : E105-E113, 2006

23. Kim DG, Jo SR, Park YS, Hyun SJ, Kim KJ, Jahng TA, et al. : Multi-channel motor evoked potential monitoring during anterior cervical discectomy and fusion. Clin Neurophysiol Pract 2 : 48-53, 2017

24. Lall RR, Lall RR, Hauptman JS, Munoz C, Cybulski GR, Koski T, et al. : Intraoperative neurophysiological monitoring in spine surgery: indications, efficacy, and role of the preoperative checklist. Neurosurg Focus 33 : E10, 2012

25. Lee JM, Kim DH, Kim HS, Choi BK, Han IH : The applicability of intraoperative neuromonitoring in patients with preoperative motor weakness 
during spine surgery. Korean J Spine $13:$ 9-12, 2016

26. Lee JY, Hilibrand AS, Lim MR, Zavatsky J, Zeiller S, Schwartz DM, et al. : Characterization of neurophysiologic alerts during anterior cervical spine surgery. Spine (Phila Pa 1976) 31 : 1916-1922, 2006

27. Ney JP, van der Goes DN, Watanabe JH : Cost-effectiveness of intraoperative neurophysiological monitoring for spinal surgeries: beginning steps. Clin Neurophysiol 123 : 1705-1707, 2012

28. Nuwer MR, Emerson RG, Galloway G, Legatt AD, Lopez J, Minahan R, et al. : Evidence-based guideline update: intraoperative spinal monitoring with somatosensory and transcranial electrical motor evoked potentials: report of the Therapeutics and Technology Assessment Subcommittee of the American Academy of Neurology and the American Clinical Neurophysiology Society. Neurology 78 : 585-589, 2012

29. Park MK, Lee SJ, Kim SB, Lee KW, Lee HJ, Han EY, et al. : The effect of positive changes during intraoperative monitoring of the functional improvement in patients with cervical compressive myelopathy. Clin Interv Aging $13: 1211-1218,2018$

30. Rajshekhar V, Velayutham P, Joseph M, Babu KS : Factors predicting the feasibility of monitoring lower-limb muscle motor evoked potentials in patients undergoing excision of spinal cord tumors. J Neurosurg Spine $14: 748-753,2011$

31. Sala F, Palandri G, Basso E, Lanteri P, Deletis V, Faccioli F, et al. : Motor evoked potential monitoring improves outcome after surgery for intramedullary spinal cord tumors: a historical control study. Neurosurgery 58 : 1129-1143; discussion 1129-1143, 2006

32. Smith PN, Balzer JR, Khan MH, Davis RA, Crammond D, Welch WC, et al. : Intraoperative somatosensory evoked potential monitoring during anterior cervical discectomy and fusion in nonmyelopathic patients--a review of 1,039 cases. Spine J $7:$ :83-87, 2007

33. Taunt CJ Jr, Sidhu KS, Andrew SA : Somatosensory evoked potential monitoring during anterior cervical discectomy and fusion. Spine (Phila Pa 1976) 30 : 1970-1972, 2005

34. Traynelis VC, Abode-lyamah KO, Leick KM, Bender SM, Greenlee JD : Cervical decompression and reconstruction without intraoperative neurophysiological monitoring. J Neurosurg Spine 16 : 107-113, 2012

35. Uchida K, Nakajima H, Sato R, Kokubo Y, Yayama T, Kobayashi S, et al. : Multivariate analysis of the neurological outcome of surgery for cervical compressive myelopathy. J Orthop Sci 10 : 564-573, 2005

36. Uchida K, Nakajima H, Takeura N, Yayama T, Guerrero AR, Yoshida A, et al. : Prognostic value of changes in spinal cord signal intensity on magnetic resonance imaging in patients with cervical compressive myelopathy. Spine J 14 : 1601-1610, 2014

37. Wang S, Tian Y, Wang C, Lu X, Zhuang Q, Peng H, et al. : Prognostic value of intraoperative MEP signal improvement during surgical treatment of cervical compressive myelopathy. Eur Spine J 25 : 1875-1880, 2016

38. Wei L, Wei Y, Tian Y, Cao P, Yuan W : Does three-grade classification of T2-weighted increased signal intensity reflect the severity of myelopathy and surgical outcomes in patients with cervical compressive myelopathy? A systematic review and meta-analysis. Neurosurg Rev 43 : $967-$ 976, 2020

39. Xu R, Ritzl EK, Sait M, Sciubba DM, Wolinsky JP, Witham TF, et al. : A role for motor and somatosensory evoked potentials during anterior cervical discectomy and fusion for patients without myelopathy: analysis of 57 consecutive cases. Surg Neurol Int 2 : 133, 2011

40. Zileli M, Borkar SA, Sinha S, Reinas R, Alves ÓL, Kim SH, et al. : Cervical spondylotic myelopathy: natural course and the value of diagnostic techniques -WFNS Spine Committee Recommendations. Neurospine $16: 386-402,2019$ 